\title{
Deciding Where to Attend: Priming of Pop-Out Drives Target Selection
}

\author{
Jan W. Brascamp, \\ Department of Psychology, Vanderbilt University \\ Randolph Blake, and \\ Sciences, Seoul National University, Seoul, Korea \\ Árni Kristjánsson \\ Department of Psychology, University of Iceland, Reykjavik, Iceland
}

Department of Psychology, Vanderbilt University, and Department of Brain and Cognitive

\section{Abstract}

With attention and eye-movements humans orient to targets of interest. This orienting occurs faster when the same target repeats: priming of pop-out (PoP). While reaction times (RTs) can be important, PoP's real function could be to steer where to orient, a possibility underexposed in many current paradigms, as these predesignate a target to which to orient. In a novel procedure we intermixed pop-out trials (one oddball target, two identical distractors) with choice trials (one item of each kind) where observers freely chose an item to attend to. Pop-out trials strongly drove subsequent choice: observers typically chose the preceding target. Conversely, choice trials affected subsequent pop-out RTs. Conventional PoP measures correlated positively with our choice measures among observers, suggesting common mechanisms. Our results support PoP accounts centered on altered target priority, and underscore PoP's importance for visual exploration.

\section{Keywords}

attention; priming; visual search; attentional selection; pop-out search

Humans move their eyes about three times each second (Rayner, 1998), yet we can easily go for days (hundreds of thousands of eye movements) without giving any thought to the question where to look next. Similar automaticity characterizes covert attention shifts made without accompanying eye movements. These facts are especially remarkable, given the vital importance of these covert and overt attention shifts, in a visual world that contains more information than we can ever hope to process. Our visual system relies, not on inordinate processing capacity, but on an exquisite ability to dedicate its limited capacity to the right input (Chun \& Nakayama, 2010; Kristjánsson \& Campana, 2010). How does the visual system achieve this?

In priming of pop-out ( $\mathrm{PoP})$ observers respond to a pop-out target presented among distractors (e.g., a red item among green distractors), in a task that requires directing attention (Maljkovic \& Nakayama, 1994; Maljkovic \& Nakayama 1996) or gaze (Bichot \& 
Schall, 2002; McPeek, Majkikovic, \& Nakayama, 1999) to the target. When the same target and distractor features repeat, response times are strongly reduced, compared to trials where the target and distractors reverse roles (Kristjánsson \& Driver, 2008; Lamy, Antebi, Aviani, \& Carmel, 2008). Several ecological interpretations of PoP suggest that it could aid visual exploration by facilitated orienting to elements important for behavior in the recent past (Chun \& Nakayama, 2010; Kristjánsson, 2006; Maljkovic \& Nakayama, 2000).

Remarkably, however, the typical PoP paradigm does not actually test directly whether PoP influences where people orient next. The correct target on each trial is instead predetermined and experimenters measure how fast observers respond to this item, rather than leaving target choice to the observer and measuring which item they select.

Existing conceptualizations of PoP vary in the degree to which they would predict an influence of PoP on the choice of where to orient. Some conceptualizations build on the notion of a "salience map" (Fecteau \& Munoz, 2006; Itti \& Koch, 2001; Nothdurft, 2010; Wolfe, 1994), construed as a neural representation of the relative priorities of display elements that guides attention and eye movements so that the element of highest priority is selected. According to these conceptualizations the observed RT benefit for selecting a repeating target item stems from the increased priority of that item which arises when it is first selected, as well as from a decrease in the priority of the distractors. Together, these influences shorten the time required for selecting the same item again (Becker, 2008; Fecteau \& Munoz, 2003; Findlay \& Gilchrist, 2005; Lee, Mozer, \& Vecera, 2009). Because, by definition, the item with the highest priority in the salience map tends to be selected, it is reasonable to infer on the basis of such conceptualizations that the same process could also influence the choice of which item to select, even if RT benefits do not show this directly. Some empirical support for this inference comes from eye movements studies: during popout trials where oddball and distractor features are switched relative to the previous trial observers make more erroneous eye movements to distractors, presumably because distractors have a relatively high priority on those trials (Becker, 2008; Becker \& Horstman, 2009; Bichot \& Schall, 2002; McPeek et al., 1999).

Other conceptualizations, however, would not necessarily predict that PoP would influence the choice of where to orient. For instance, Hillstrom (2000) proposes that PoP influences how fast the priorities of display elements are determined, yet without altering the priorities themselves. Such a process would result in RT changes in selecting an item, but it does not follow that the choice of which item to select would also be affected, because this is determined by the items' priorities, which remain unchanged. Lamy, Carmel, Egeth, and Leber (2006), in turn, suggest that PoP influences how quickly attention (or gaze) is directed to an item, but only after the item has already been selected as a target. Again, given that this scenario would place the effect of PoP downstream of the selection process itself, the outcome of this selection process should remain unaltered and only the RT should change. We will address the empirical support for these conceptualizations in the Discussion section.

The aim of the current study was to assess the role that PoP plays in target selection. We introduce a new procedure that directly assesses target choice by interspersing pop-out trials with free-choice trials where there is no designated target and observers choose on which item to report (see Figure 1).

\section{Experiment 1: Pop-Out Influences Subsequent Target Choice}

In Experiment 1 we investigated the role PoP plays in determining where to direct attention. On every trial observers viewed a set of diamonds, each either red or green and each with one of its four corners cut off (see Figure 1). Observers reported which corner of one of the diamonds had a notch, a task requiring focal attention. On standard pop-out trials, the target 
item for report was defined by having a different color than the other two items, termed distractors (Figure 1A). As a novel aspect of our approach, we intermixed "choice" trials (Figure 1B), where there was no predesignated target. On those trials the observers were simply asked to perform the notch task on either one of two presented diamonds, one of each color, leaving target choice to the observer.

\section{Method}

Colored diamonds ( $2 \mathrm{deg}$ diameter) were presented on a black background $\left(0.22 \mathrm{~cd} / \mathrm{m}^{2}\right)$ on an imaginary ring ( $8 \mathrm{deg}$ radius) around a white fixation spot $\left(86 \mathrm{~cd} / \mathrm{m}^{2}\right)$. The diamonds occupied random, yet equally spaced positions on the ring (at 120 or 180 degree intervals on pop-out trials and choice trials, respectively). Red luminance was $18.1 \mathrm{~cd} / \mathrm{m}^{2}$, and each observer's subjectively equivalent green luminance was determined using flicker photometry (mean $17.7 \mathrm{~cd} / \mathrm{m}^{2}$ ). Notch sides were chosen randomly, but each diamond on a given trial had a notch at a different corner. This enabled us to identify the elected target on choice trials by the reported notch side. ${ }^{1}$ Seven naive observers reported the notch side of the target diamond using their dominant hand on four keyboard keys, while keeping fixation. They were asked to respond as quickly as possible while maintaining a low error rate. Observers were informed beforehand that trials with three items and a designated target would form the majority of trials, with trials with only two items making up a smaller proportion. Observers were also told that, when there were only two items, they were entirely free to select whichever one they wanted, neither being wrong. Stimuli stayed on the screen until response, and following a random delay (1.5 to $2 \mathrm{~s}$ ) a new trial started. Pop-out trials appeared in runs of repeating target color. One-third of these runs were followed by a run of opposite target color; the remaining two-thirds were followed by a single choice trial. Each choice trial was again followed by a pop-out run of random target color. Pop-out runs lasted 1,3 , or 6 trials, randomly selected. This yielded about 700 pop-out trials and 60 choice trials per session.

\section{Results and Discussion}

RTs on pop-out trials (excluding 4\% incorrect responses) revealed conventional PoP (Figure 2A). RTs were shorter when pop-out target color repeated relative to the preceding pop-out trial $(M=682 \mathrm{~ms})$ than when it switched $(M=724 \mathrm{~ms}$; two-tailed paired $t$ test $t(\sigma)=3.74, p$ $<.01)$. Also, RTs progressively shortened across pop-out trials within a run of repeating target color (dark curve in Figure 2A; repeated-measures ANOVA with trial number as an across-subject factor, $F(4,24)=7.1, p<.001$; Spearman test on individual observers, $\rho=$. 21 , two-sided $p<.01)$. Conversely, RTs on pop-out trials progressively increased as the length of an immediately preceding run of opposite color pop-out targets was longer (light curve in Figure 2A; repeated-measures ANOVA with trial number as an across-subject factor, $\mathrm{F}(2,12)=7.5, p<.01$; Spearman test on individual observers, $\rho=.34$, two-sided $p$ $<.001)$. This confirms that our paradigm induced the standard RT patterns that characterize PoP.

Figures $2 \mathrm{~B}$ and $2 \mathrm{C}$ show across-observer results on choice trials, discounting error responses. ${ }^{1}$ Every choice trial was preceded by one, three or six consecutive pop-out trials with a given target color. A single pop-out trial (Figure 2B, leftmost bars) strongly biased subsequent target choice, with $68 \%$ of choices for the diamond with the earlier pop-out color

\footnotetext{
${ }^{1}$ On $2 \%$ of choice trials observers reported a corner where neither diamond had a notch. These trials were rejected. This low percentage confirms that we can reliably infer target choice from reported cut-off side: on choice trials where observers make a mistake reporting the cut-off side of their selected target, their response is expected to correspond to neither diamond $2 / 3$ of the time, and to the nonselected diamond $1 / 3$ of the time (there being four possible reports in total). The $2 \%$ figure thus indicates that occasions where an observer's report caused us to incorrectly infer which diamond was chosen amounted to only about $1 \%$ of the trials.
} 
(two-tailed $t$ test testing against 50\%, $t(\sigma)=3.7, p<.01$ ). This bias increased with the length of the pop-out run (Figure 2B, repeated-measures ANOVA with run length as an acrosssubject factor, $F(2,12)=12.4, p<.01$; Spearman test on individual observers, $\rho=.60$, twosided $P<.01)$. Following six-trial pop-out runs, target choice was almost fully determined, with observers choosing the prior pop-out color $88 \%$ of the time.

To further characterize the effects of trial history on the process of selecting a target, Figure $2 \mathrm{C}$ shows RTs on choice trials. Trials where observers chose a target that repeated the preceding pop-out color yielded faster RTs than trials where they did not (repeated-measures ANOVA with across-subject factors "color repeat" vs. "color switch" and run length, $F(1,6)$ $=44.2, p<.001$ ) Like the bias in target choice itself (Figure 2B), this RT effect increased with preceding run length (same ANOVA, $F(2,12)=19.7, p<.001$; Spearman test on the difference in RTs for individual observers, $\rho=.58$, two-sided $p<.05$ ).

\section{Experiment 2: Target Choice Is Still Affected When Targets Are Unpredictable}

We next asked whether the pattern of results of Experiment 1 depends on observers' explicit expectations of upcoming trials, or whether they can arise independent of conscious anticipation. Considering that directing attention and gaze in daily life usually do not involve conscious planning, a potential role of the present priming effects in these processes requires that these effects occur automatically. Note that "automatic" here does not mean that conscious effort could not interfere with target choice in principle, but only that it does not account for the pattern of results here. To be sure, one can consciously decide where to look if so inclined, even though most eye movements proceed without such explicit decisions. In other words, by "automatic" we mean that the priming process occurs, at least in part, outside of conscious awareness.

In Experiment 1 we showed that both choice bias and RT change progressively as a function of the number of preceding repetitions of the same target color (see Figure 2). This suggests that explicit strategies do not account for the observed results because observers are generally unable to explicitly recall target color further than about one trial back (Maljkovic \& Nakayama, 2000). Moreover, it is known that conventional PoP does not rely on conscious strategies, and even occurs when it is pitted against observers' intentions (Maljkovic \& Nakayama, 1994, Experiment 4; see also Kristjánsson \& Nakayama, 2003). Still, the design of our Experiment 1 differed from the designs used in several existing studies, in that target color in Experiment 1 remained constant and, in principle, predictable during streaks of several repetitions, rendering a role of explicit expectation more plausible. We, therefore, repeated Experiment 1 while randomly varying target color from trial to trial, to determine whether this unpredictability would eliminate the sequential RT effects and choice bias effects seen in Figure 2.

\section{Method}

We used the same stimuli as Experiment 1 but now single choice trials were alternated with PoP trial sequences that could be either 3 , 4, or 5 trials in length. This length was determined randomly for each sequence. This yielded about 600 pop-out trials and 150 choice trials per session. Target color was randomly set to either red or green on each individual PoP trial. The reason for choosing three as the minimum length of a sequence of PoP trials was that we wanted to efficiently measure priming effects accumulated during up to three preceding PoP trials, and we randomly varied the number of PoP trials per sequence so that the occurrence of a choice trial would be unpredictable. Six naive observers participated, none 
of whom had previously run in Experiment 1. Instructions were the same as in Experiment 1.

\section{Results and Discussion}

Figure 3 shows across-observer averaged results on choice trials, discounting error responses (3\% of trials). As in Experiment 1, observers were more likely to choose the target that had the same color as the preceding pop-out target (Figure 3A). This effect was already significant after a single pop-out target of a given color $(62 \%$ chance of choosing the preceding target color; two-tailed $t$ test testing against $50 \%, t(5)=3.01, p<.05)$ and it grew as the number of preceding same-color pop-out targets increased (repeated-measures ANOVA with run length as an across-subject factor, $F(2,10)=14.7, p<.01$; Spearman test on individual observers, $\rho=.43$, two-sided $p<.05$ ).

As in Experiment 1, RTs were significantly shorter on choice trials when observers selected the diamond that shared the preceding pop-out target's color, and this effect grew stronger with the length of the preceding same-color pop-out run (Figure 3B; a repeated-measures ANOVA with across-subject factors "color repeat" vs. "color switch" and run length shows a main effect of color repetition, $F(1,5)=21.7, p<.1$, as well as an interaction between color repetition and run length, $F(2,10)=17.3, \mathrm{p}<.01$. A Spearman test on the difference in RTs for individual observers confirms the effect of run length: $\rho=.55$, two-sided $p<.05$ ).

In sum, Experiment 2 replicates the effects of trial history observed in Experiment 1 using a randomized design that minimizes a potential role of explicit expectations. This indicates that the priming effects we observe can arise in the absence of explicit strategies.

\section{Reanalysis of Experiments 1 and 2: Correlations Between Classic PoP and Choice Effects}

The above results show how pop-out trials influence subsequent target choice. Do these choice effects reflect the same priming mechanisms which result in RT benefits in conventional PoP, or are these unrelated phenomena? In principle, it is possible that these choice effects show a form of priming that occurs alongside standard PoP effects, while drawing on different mechanisms. To clarify this issue we investigated whether individual observers in Experiments 1 and 2 who experienced larger RT benefits in conventional PoP also tended to display a larger effect of preceding pop-out trials on subsequent target choice. If these two effects have an overlapping neural substrate, such a correlation is predicted.

\section{Method}

For each observer we quantified their conventional PoP effect as the difference between their average RT on pop-out trials that followed a pop-out trial with the same target color as the current trial, and their average RT on pop-out trials that followed a pop-out trial with the opposite target color. Analogously, the effect of trial history on target choice was quantified as the probability that an observer chose that same color as on the immediately preceding pop-out trial.

\section{Results}

Figure 4 shows the result of this analysis, with each dot representing a single observer. Both in Experiment 1 (panel A) and in Experiment 2 (panel B) there was a significant positive correlation between interobserver variation in the strength of target choice effects and the strength of conventional PoP effects (Spearman test, $\rho=.82$, two-sided $p<.05$ for 
Experiment 1, and $\rho=1$, two-sided $p<.01$ for Experiment 2). Recall that there was no overlap between these experiments' observer pools, implying that this is a general result. For a fairer interobserver comparison Figure 4 expresses RT effects as a proportion of each individual observer's average RT; however, we find similar correlations regardless of this normalization step. These results show that observers differ in a correlated fashion in both the strength of conventional PoP and the strength of the choice effects we describe, consistent with the idea that the two phenomena have at least partially shared underlying mechanisms.

\section{Experiment 3: Target Choice Induces RT Benefits}

If conventional PoP and our present choice effects are indeed two manifestations of the same cognitive operations, then it should be possible to turn Experiments 1 and 2 around, asking whether target selection on free choice trials influences subsequent RT on pop-out trials. In other words: Do free choice trials result in the RT benefits that characterize conventional PoP, or does PoP only build up if the target is predetermined?

\section{Method}

Single pop-out trials (with random target color) were interspersed between longer runs of 4 , 6 , or 8 choice trials each (randomly selected). This strongly increased the proportion of choice trials, to about 650 choice trials and 100 pop-out trials per session. Ten naive observers reported the notch position on the oddball diamond on pop-out trials, and on a freely selected diamond on choice trials. Six of these observers had previously participated in Experiment 1, but all our results remain qualitatively the same when analyzing only the four observers who had not (data not shown). Instructions were the same as in the previous experiments, but observers were notified that free-choice trials would make up the majority of trials this time.

\section{Results and Discussion}

All observers were balanced in the color of their chosen target on choice trials (average imbalance 57\% / 43\%), indicating no overall color preference. More interestingly, RTs on pop-out trials were faster when target color was the same as on the immediately preceding choice trial than when it switched (Figure 5; two-tailed paired $t$ test, $t(9)=2.9, p<.05$ ). Moreover, this effect of choice trials on subsequent pop-out RT depended on run length; that is, on the number of consecutive choice trials on which a given-color target was chosen preceding the current pop-out trial (Figure 5; repeated-measures ANOVA with run length and same vs. opposite color as across-subject factors, interaction between both factors $F(3$, $27)=152, p<.001$; a Spearman test on individual observers' differences between mean "same" RT and mean "opposite" RT falls just short of significant, at $\rho=.30$, two-sided $p=$. $06)$.

Compared to conventional PoP that results from preceding pop-out trials, the effect of preceding choice trials in Figure 5 is both qualitatively similar and similar in size (cf Figures 2 and 3) which supports the notion that the robust effect associated with free choices arises from the same process that underlies conventional PoP. Evidently, the buildup of PoP does not require a pop-out target; the mere selection of a target suffices to induce PoP.

\section{General Discussion}

We introduced a new procedure to study attentional priming, where target choice, rather than RT, is the main dependent variable. Our results indicate that priming can strongly influence what observers attend to (Figures 2 and 3), and that priming develops whenever 
elements receive attention, even without a pop-out target (see Figure 5). These results underscore the importance of priming in automatically guiding where to orient during natural vision (Chun \& Nakayama, 2010; Kristjánsson \& Campana, 2010; Maljkovic \& Nakayama, 2000), along with other factors such as feature contrast and top-down control (Findlay \& Gilchrist, 2005; Itti \& Koch, 2001; Wang, Kristjánsson, Nakayama, 2005; Wolfe $\&$ Horowitz, 2004). The fact that the effect of priming on target choice and conventional PoP are correlated on an observer-to-observer basis (see Figure 4) supports the notion that traditional PoP paradigms probe mechanisms that overlap, partially or fully, with the mechanisms that cause altered target choice in our experiments, consistent with fMRI findings that $\mathrm{PoP}$ is associated with activation in brain areas related to attention deployment (Kristjánsson, Vuilleumier, Schwartz, Macaluso, \& Driver 2007). Our results are consistent with the view that, like conventional PoP, choice priming occurs automatically in the sense that no conscious recollection or strategies are required. The implicit, yet powerful, nature of the effect of priming on target choice is highlighted by our observers' subjective impressions during choice trials. One mentioned "a clear intuition which one to choose, with hardly ever any doubt," and another found himself "already attending to one diamond, only then to realize that there was no pop-out target."

Our results highlight the value of free choice designs for probing attention allocation, a value also indicated by previous work where observers freely inspected a display for about a second and were then asked which items they had noticed (Pashler \& Harris, 2001). Free choice designs complement experiments that focus on reaction time and have the benefit of staying close to natural situations, where scenes are often cluttered with many elements, none of which counts as a "target".

We have consistently interpreted changes in the reported target following priming as changes in attention allocation. It is worth noting that we never measured attention allocation directly (e.g., in terms of performance on a visual task) and that notch report is an indirect measure of attention allocation. Nevertheless, we believe it is reasonable to infer from our results that priming influences attentional selection. An alternative scenario, that priming leaves attention allocation unaffected but instead modulates observers' decision of whether to report on an attended item, would predict that priming would increase the number of trials where observers first attend to the unprimed item without reporting its notch, before redirecting attention to the other item and providing a report. This would result in increased reaction times associated with stronger priming, but we found that reaction times were actually reduced, arguing against this possibility (see Figure 2). This supports the notion that our experiments indeed measured the effects of priming on attentional selection.

Our results do not distinguish between whether priming effects arise from a reduced potential to attract attention on the part of the distractors or from an increased potential of the target. In our design target and distractor colors could either stay the same or switch roles, so such distractor inhibition and target facilitation would always co-occur. Other studies have shown that both effects, in fact, contribute jointly to conventional PoP (Geyer, Ze-hetleitner, \& Muller, 2010; Kristjánsson \& Driver, 2008; Lamy et al., 2008;Wang et al., 2005). It, therefore, seems likely that our results reflect a joint outcome of these two factors as well, but further research would be required to verify this.

Our results support and extend findings from eye movement studies showing that the identity of the target on previous trials influences which items observers foveate during the current trial before fixating on the target (Becker, 2008; Becker \& Horstman, 2009; Bichot \& Schall, 2002; McPeek et al., 1999). In those studies effects of trial history on selection behavior were evident in increased numbers of erroneous saccades to nontargets during trials where target features switched relative to the preceding trial, compared to when they 
stayed the same. Here we extend these findings and demonstrate that priming of attentional selection shows similar patterns to priming of eye movements.

One question that arises from this work regards the finding that free choice trials leave a similar priming trace as pop-out trials (see Figure 5). This indicates that endogenous attention allocation on choice trials and partly exogenous attention allocation on pop-out trials do not differ strongly in their influence on how attention is subsequently distributed. An interesting objective for future work would be to see if this same conclusion holds when the pop-out target is made more salient, so that the balance during pop-out trials is shifted even more toward exogenous attention. One indication that this shift would, in fact, reduce the strength of priming is that increasing distractor numbers, which increases the oddball target's salience, reduces priming strength (Geyer et al., 2010).

This study was in part motivated by work on implicit memory for ambiguous stimuli: the tendency to perceive a reappearing ambiguous image in the same way it was perceived before (Pearson \& Brascamp, 2008). Whereas many mnemonic functions differ from this "perceptual memory" in elementary properties such as their timescale or whether conscious recollection is involved, PoP stands out by sharing its implicit nature, its timescale and the ability to accumulate across trials (Brascamp et al., 2008), along with more general parallels between attentional selection and ambiguous perception (Kristjánsson, 2009; Leopold \& Logothetis, 1999; Mitchell, Stoner, \& Reynolds, 2004). One apparent difference was that "perceptual memory" alters the (perceptual) choice between two percepts, whereas PoP entails an RT benefit. The present work, however, indicates that PoP, similarly, alters (attentional) choice. Moreover, the buildup of priming during trials without a predesignated target (see Figure 5) indicates that priming arises from internal choices, regardless of external confirmation. This is also seen for "perceptual memory," where neither perception is "correct". Our work thus lends credence to the idea of a potential relation between these two functions.

Our results have implications for theories of PoP, arguing against conceptualizations which assume that PoP does not affect target selection directly. Specifically, Hillstrom (2000) proposed that PoP does not change the priorities of display elements that determine where attention is allocated, instead attributing the RT effects to changes in the speed at which those priorities are determined. Without modification this idea cannot explain our findings, because unaltered priorities would mean unaltered target selection on free choice trials, at odds with our findings (Figures 2 and 3). On the same grounds, our results are inconsistent with the suggestion that PoP may act downstream of the point where a target is selected, modifying the speed at which attention is subsequently allocated to that target (Lamy et al., 2006).

Given the present evidence that PoP biases target selection, it is important to recall what empirical findings gave rise to the notion that it does not. Hillstrom (2000) reached the conclusion that search priorities are unaltered following priming based on the observation that repeating target features in a conjunction search task reduced search times but did not alter the "search function" curve relating search time to the number of distractors in the display. The slope of the search function is thought to depend, in part, on how many distractors the observer attends to before selecting the target: the larger the number of attention allocations to distractors, the steeper the search function. This interpretation of the search function underlies the view that an observed lack of effect of target feature repetition on the slope of the search function indicates a lack of effect on target priority, because increased target priority would be expected to reduce the probability of orienting to distractors instead (Hillstrom, 2000). One possibility is that priming effects are manifested in the shape of the search function after all, yet that this is not evident in all experimental 
designs due to, for instance, statistical power. Indeed, more recent work has shown the expected effects of priming on the search function (Becker \& Horstmann, 2009). In addition, that same study discusses reasons why the shape of the search function may not always be the most sensitive measure of changes in attentional priorities, one potential reason being that experimental manipulations such as priming, in addition to affecting priorities, may have other effects that also influence the search function. In that case more pure and direct measures of attentional priority, such as selection behavior on our choice trials, are preferable.

Lamy et al. (2006) based their argument that PoP may not influence target priority on the observation that repeating target features reduced RTs in an oddball search task but did not reduce interference from an irrelevant oddball distractor that was randomly included on some trials, as measured by RT. Their reasoning is similar to the one above: If priming increases the priority, or salience, of the target, then an irrelevant distractor should have a reduced potency of attracting attention at the target's expense, leading to reduced interference. One option is that priming may in principle reduce distractor interference, but that this effect was small in the Lamy et al. study and, therefore, overlooked. Particularly, the use of a highly salient color singleton as an irrelevant distractor may have rendered priming-related modulations of the salience of the shape-defined target modest in comparison, and an effect on distractor interference may surface in experiments that use a less powerful distractor. An indication that this may be the case is that the RT cost of distractor interference in the Lamy et al. study was as large or larger than the RT benefit of repeating the target features, suggesting that the oddball distractor was quite salient. It is worth noting that Pinto, Olivers, and Theeuwes (2005) and Lamy and Yashar (2008) reported exactly the reduction in distractor interference through priming that one would expect if priming alters priority. This result is limited, however, to certain paradigms where distractor presence is predictable, and has not been observed when distractors are included unpredictably (Lamy \& Yashar, 2008; Lamy et al., 2006).

Our results fit well with the notion that target selection is based on relative priorities of display elements, such as those laid out in "salience maps" (Fecteau \& Munoz, 2006; Itti \& Koch, 2001; Nothdurft, 2010; Wolfe, 1994), and that selection history alters these priorities (Becker, 2008; Fecteau \& Munoz, 2003; Lee et al., 2009). On pop-out trials the goal of selecting the oddball largely dictates where to attend, and history effects are restricted to RTs. On choice trials no such goal is provided, and priming becomes the dominant determinant of target selection, pointing to a similarly important role in natural exploration.

\section{Acknowledgments}

This work was supported by a United States National Institutes of Health Core Grant (P30-EY008126; RB), by a Rubicon grant from the Netherlands Organisation for Scientific Research (JB), by a WCU program through the National Research Foundation of Korea funded by the Ministry of Education, Science and Technology (RB), and by funding from the Research Fund of the University of Iceland (AK).

\section{References}

Becker S. The mechanism of priming: Episodic retrieval or priming of pop-out? Acta Psychologies. 2008; 127:324-339.10.1016/j.actpsy.2007.07.005

Becker S, Horstmann G. A feature-weighting account of priming in conjunction search. Attention, Perception \& Psychophysics. 2009; 71:258-272.10.3758/APP.71.2.258

Bichot N, Schall J. Priming in macaque frontal cortex during pop-out visual search: Feature-based facilitation and location-based inhibition of return. The Journal of Neuroscience. 2002; 22:46754685. [PubMed: 12040074] 
Brascamp J, Knapen T, Kanai R, Noest A, van Ee R, van den Berg A. Multi-timescale perceptual history resolves visual ambiguity. PLoS ONE. 2008; 3:e1497.10.1371/journal.pone.0001497 [PubMed: 18231584]

Chun M, Nakayama K. On the functional role of implicit visual memory for the adaptive deployment of attention across scenes. Visual Cognition. 2010; 7:65-81.10.1080/135062800394685

Fecteau J, Munoz D. Exploring the consequences of the previous trial. Nature Reviews Neuroscience. 2003; 4:435-443.10.1038/nrnll14

Fecteau J, Munoz D. Salience, relevance, and firing: A priority map for target selection. Trends in Cognitive Sciences. 2006; 10:382-390.10.1016/j.tics.2006.06.011 [PubMed: 16843702]

Findlay, JM.; Gilchrist, ID. Eye guidance and visual search. In: Underwood, G., editor. Cognitive processes in eye guidance. New York, NY: Oxford University Press; 2005. p. 259-282.

Geyer T, Zehetleitner M, Mülller H. Positional priming of pop-out: A relational-encoding account. Journal of Vision. 2010; 10:1-17. [PubMed: 20462304]

Hillstrom A. Repetition effects in visual search. Perception \& Psychophysics. 2000; 62:800817.10.3758/BF03206924 [PubMed: 10883586]

Itti L, Koch C. Computational modelling of visual attention. Nature Reviews Neuroscience. 2001; 2:194-203.10.1038/35058500

Kristjánsson Á. Rapid learning in attention shifts: A review. Visual Cognition. 2006; 13:324362.10.1080/13506280544000039

Kristjánsson Á. Learning in shifts of transient attention improves recognition of parts of ambiguous figure-ground displays. Journal of Vision. 2009; 9(4):1-11.

Kristjánsson A, Campana G. Where perception meets memory: A review of repetition priming in visual search tasks. Attention, Perception \& Psychophysics. 2010; 72:5-18.10.3758/APP.72.1.5

Kristjánsson Á, Driver J. Priming in visual search: Separating the effects of target repetition, distractor repetition and role-reversal. Vision Research. 2008; 48:1217-1232.10.1016/j.visres.2008.02.007 [PubMed: 18374961]

Kristjánsson Á, Vuilleumier P, Schwartz S, Macaluso, Driver J. Neural Basis for Priming of Pop-Out during Visual Search Revealed with fMRI. Cerebral Cortex. 2007; 17:1612-1624.10.1093/cercor/ bhl072 [PubMed: 16959868]

Kristjánsson Á, Nakayama K. A primitive memory system for the deployment of transient attention. Perception \& Psychophysics. 2003; 65:711-724.10.3758/BF03194808 [PubMed: 12956579]

Lamy D, Antebi C, Aviani N, Carmel T. Priming of Pop-out provides reliable measures of target activation and distractor inhibition in selective attention. Vision Research. 2008; 48:30 41.10.1016/j.visres.2007.10.009 [PubMed: 18054983]

Lamy D, Carmel T, Egeth H, Leber A. Effects of search mode and intertrial priming on singleton search. Perception \& Psychophysics. 2006; 68:919-932.10.3758/BF03193355 [PubMed: 17153188]

Lamy D, Yashar A. Intertrial target-feature changes do not lead to more distraction by singletons: Target uncertainty does. Vision Research. 2008; 48:1274-1279.10.1016/j.visres.2008.02.021 [PubMed: 18395767]

Lee H, Mozer M, Vecera S. Mechanisms of priming of pop-out: Stored representations or feature-gain modulations? Attention, Perception \& Psychophysics. 2009; 71:1059-1071.10.3758/APP. 71.5.1059

Leopold D, Logothetis N. Multistage phenomena: Changing views in perception. Trends in Cognitive Sciences. 1999; 3:254-264.10.1016/S1364-6613(99)01332-7 [PubMed: 10377540]

Maljkovic V, Nakayama K. Priming of pop-out: I. Role of features. Memory \& Cognition. 1994; 22:657-672.10.3758/BF03209251

Maljkovic V, Nakayama K. Priming of pop-out: II. The role of position. Perception and Psychophysics. 1996; 58:977-991.10.3758/BF03206826 [PubMed: 8920835]

Maljkovic V, Nakayama K. Priming of pop-out: III. A short-term implicit memoiy system beneficial for rapid target selection. Visual Cognition. 2000; 7:571-595.10.1080/135062800407202 
McPeek R, Maljkovic V, Nakayama K. Saccades require focal attention and are facilitated by a shortterm memoiy system. Vision Research. 1999; 39:1555-1566.10.1016/S0042-6989(98)00228-4 [PubMed: 10343821]

Mitchell J, Stoner G, Reynolds J. Object-based attention determines dominance in binocular rivalry. Nature. 2004; 429:410-413.10.1038/nature02584 [PubMed: 15164062]

Nothdurft H. Salience and target selection in visual search. Visual Cognition. 2010; 14:1-30.

Pashler H, Harris C. Spontaneous allocation of visual attention: dominant role of uniqueness. Psychonomic Bulletin \& Review. 2001; 8:747-752. [PubMed: 11848595]

Pearson J, Brascamp JW. Sensory memoiy for ambiguous vision. Trends in Cognitive Sciences. 2008; 12:334-341.10.1016/j.tics.2008.05.006 [PubMed: 18684661]

Pinto Y, Olivers C, Theeuwes J. Target uncertainty does not lead to more distraction by singletons: Intertrial priming does. Perception \& Psychophysics. 2005; 67:1354-1361.10.3758/BF03193640 [PubMed: 16555587]

Rayner K. Eye movements in reading and information processing: 20 years of research. Psychological Bulletin. 1998; 124:372-422.10.1037/0033-2909.124.3.372 [PubMed: 9849112]

Wang D, Kristjánsson Á, Nakayama K. Efficient visual search without top-down or bottom-up guidance. Perception \& Psychophysics. 2005; 67:239-253.10.3758/BF03206488 [PubMed: 15971688]

Wolfe JM. Guided Search 2.0. Psychonomic Bulletin \& Review. 1994; 1:202-238.10.3758/ BF03200774

Wolfe JM, Horowitz T. What attributes guide the deployment of visual attention and how do they do it? Nature Reviews Neuroscience. 2004; 5:495-501.10.1038/nrnl411 
A. Pop-out trial
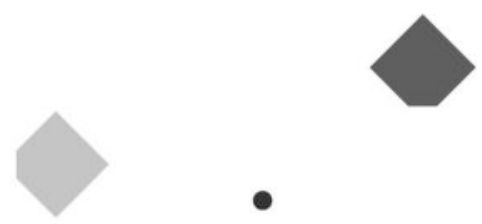

B. Choice trial

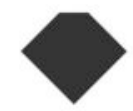

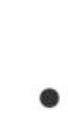

\section{Figure 1.}

Schematic depiction of our stimuli. A. On pop-out trials three diamonds were presented, and observers reported the position of the notch on the odd-colored target diamond. B. On choice trials one diamond of each color was presented, and observers freely selected a diamond for which to perform this task. 


\section{A. RT on pop-out trials}

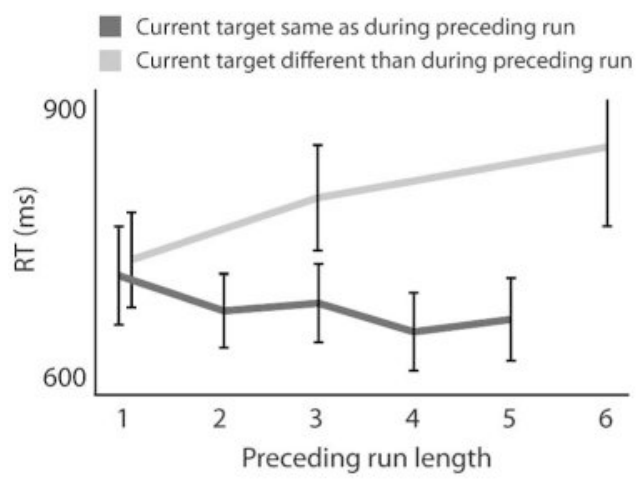

B. Target selection on choice trials

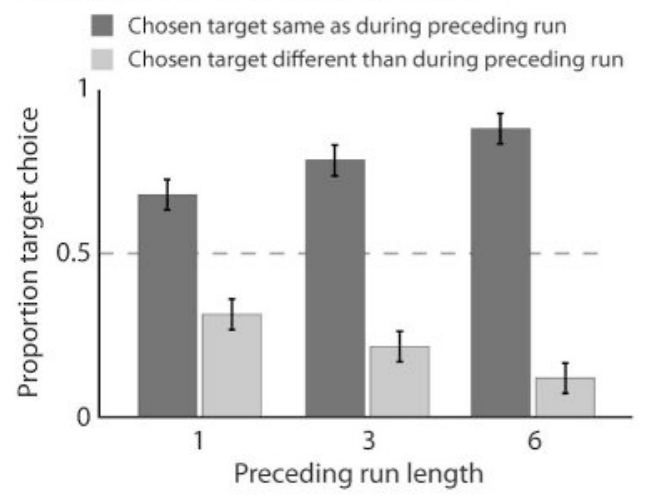

C. RT on choice trials

Chosen target same as during preceding run

Chosen target different than during preceding run

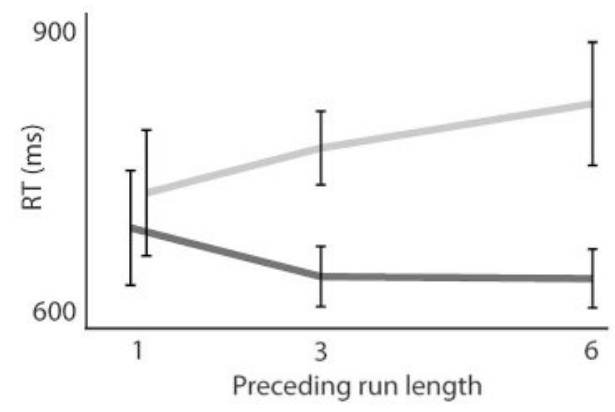

Figure 2.

Experiment 1. A. Standard PoP during pop-out trials: RT on the current trial is faster if target color is repeated relative to preceding pop-out trials. B. Target choice on choice trials (same color or different color than preceding pop-out target) following pop-out runs of various durations. Preceding pop-out targets prime this choice. C. RT on choice trials depending on whether observers chose the target or distractor color from the preceding popout trial. RTs are faster when target color is repeated. Error bars show standard errors of the sample mean (SEM). 


\section{A. Target selection on choice trials}

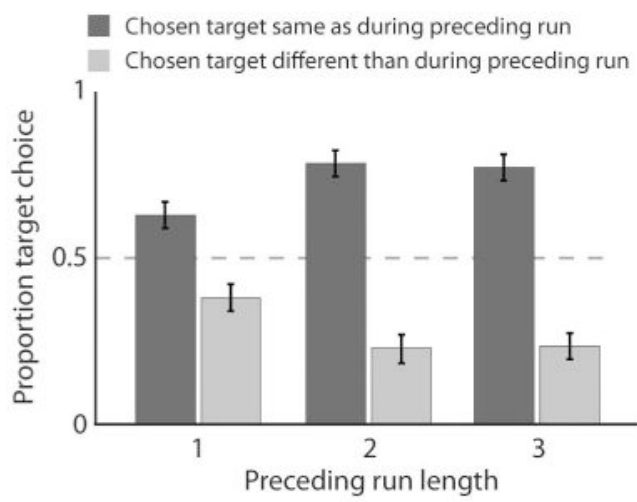

B. RT on choice trials

Chosen target different than during preceding run

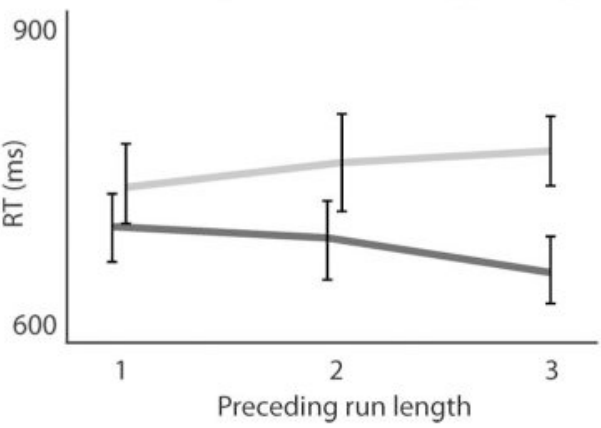

Figure 3.

Experiment 2. Replication of Experiment one using random sequences of pop-out targets. The effects of prior pop-out trials on target selection (A) and on the speed of target selection (B) are maintained in an experiment where pop-out target color varies randomly. 


\section{A. Experiment 1}

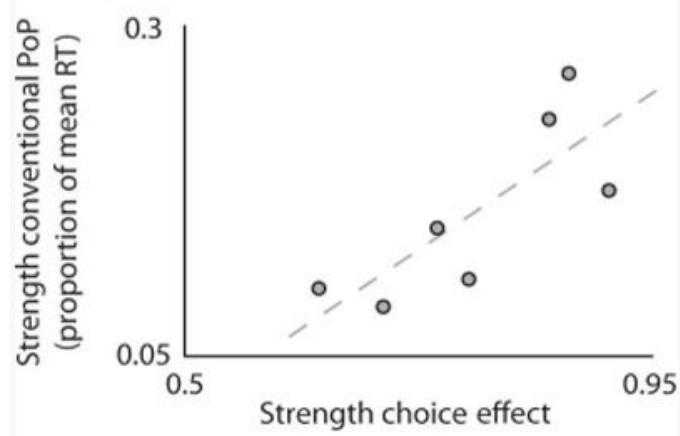

B. Experiment 2

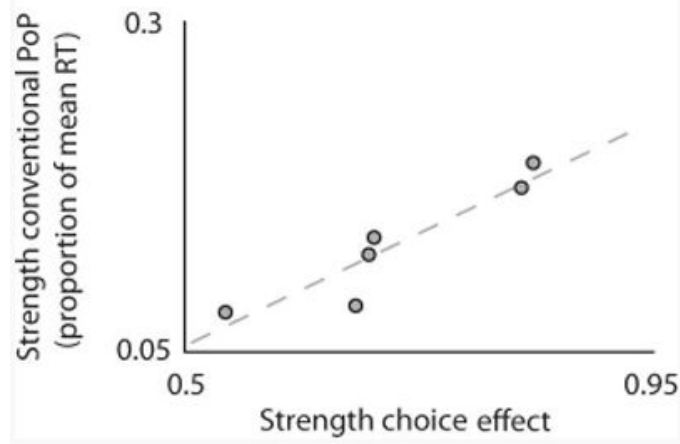

Figure 4.

Correlations between conventional PoP and choice effects. A. Interobserver variations in the extent to which target choice is biased by preceding pop-out trials is correlated with interobserver variations in the strength of conventional PoP, both in Experiment 1 (A) and in Experiment 2 (B). 


\section{RT on pop-out trials}

Target same as during preceding run of choice trials

Target different than during preceding run of choice trials

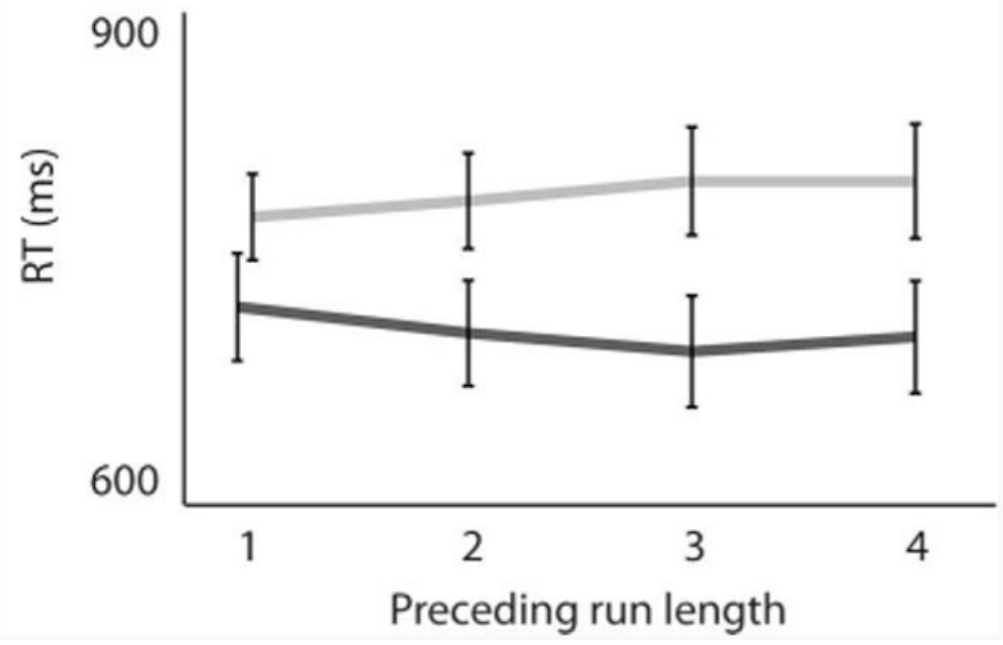

Figure 5.

Target choice affects RT on subsequent pop-out trials. Pop-out RT is shorter when target color repeats relative to preceding choice trials, an effect that accumulates across choice trials with repeated target color. 